tumor development in association with data on gene alterations, such as p53 mutations and deletion of the p16/p14ARF locus, which are hallmarks of previously defined genetic pathways. Our preliminary results with eight tumors confirm upregulation of some genes already known to be important in the development of glioblastoma and the exclusivity of some genetic pathways (p53 mutations versus EGFR overexpression). We have created expression profiles of 120 differentially expressed genes using the Cluster program from Stanford University, and we have grouped tumors according to genetic pathways. We will link patterns of differential gene expression to clinical outcomes to identify subtypes of tumors with distinct clinical behavior.

Helou, Khalil

\section{The Hgfr/Met oncogene is the target for gene amplification in DMBA-induced rat sarcomas}

\author{
Khalil Helou ${ }^{1}$, Anna Walentinsson ${ }^{1}$, Marija Kost-Alimova ${ }^{2} \&$ \\ Göran Levan ${ }^{1}$ \\ ${ }^{1}$ CMB-Genetics, Göteborg University, Göteborg, Sweden \\ ${ }^{2}$ MTC, Karolinska Institute, Stockholm, Sweden
}

Analysis of chromosome rearrangements in tumors is an important means for revealing genetic pathways underlying tumorigenesis and tumor progression. In a set of 17 rat sarcomas induced by exposure to 7,12-dimethylbenz[a] anthracene (DMBA), we had previously found homogeneously staining regions (which are generally accepted as cytogenetic signs of gene amplification) in 5 tumors, using cytogenetic analysis. By employing comparative genomic hybridization, we detected regional increases in DNA copy number of the proximal part of rat chromosome 4 (RNO4) in the tumors harboring homogeneously staining regions. We detected amplification of the $\mathrm{Hgfr} / \mathrm{Met}$ oncogene, located at RNO4q21.2, by fluorescence in situ hybridization (FISH) in all five tumors. In four of them, several flanking genes located in the near vicinity of $\mathrm{Hgfr} / \mathrm{Met}$, including Cav1 (q21.1), Wnt2 (q21.2? 21.3 ) and Cftr (q21.3), were also amplified, although amplification was seen in a smaller fraction of the cells than $\mathrm{Hgfr} / \mathrm{Met}$ amplification. In the fifth tumor (BL150T), Hgfr/Met was amplified in all cells and was the sole amplified gene of those tested. In addition the $\mathrm{Hgfr} / \mathrm{Met}$ FISH signals in BL150T were tightly clustered and formed compact and intense spots compared with the signals seen in the other four tumors. Application of the free chromatin FISH technique to BL150T showed that the genomic $\mathrm{Hg} f r / \mathrm{Met}$ probe stained the extended chromatin fibers of up to 1.5 megabases with an almost uninterrupted signal, indicating that the BL150T amplicon was build up solely from $\mathrm{Hgfr} / \mathrm{Met}$ gene sequences. Our results indicate that the $\mathrm{Hgfr} / \mathrm{Met}$ oncogene may be the primary target for amplification in a subset of rat DMBA sarcomas.

Hermann, Thomas

\section{Effects of the RXR-specific ligand Targretin on gene expression in carcinogen-induced mammary tumors in the rat}

\author{
Thomas Hermann, Patricia Tooker, Martin Seidel \\ $\&$ William Lamph
}

Ligand Pharmaceuticals, 10275 Science Center Drive, San Diego, California 92121, USA

Targretin (LGD1069), a high-affinity ligand for the retinoid X receptors (RXRa, $\mathrm{RXRb}$ and RXRg), is highly effective in the prevention and treatment of carcinogeninduced breast cancer in various rat models. To understand the molecular basis of the tumor response to Targretin, we used microarray analysis to compare the gene expression profiles of tumors regressing in response to Targretin with those of either vehicle-treated tumors or tumors resisting Targretin treatment. The vehicletreated and Targretin-resistant tumors had very similar profiles, but the tumors responding to Targretin exhibited broad changes in gene expression. These included genes involved in proliferation, markers of differentiation, tumor markers and expressed sequence tags of unknown function. We selected a subset of these genes for further evaluation. Quantitative analysis by real-time polymerase chain reaction confirmed the differential expression for the majority of the selected genes. However, a small fraction was identified as false positives. We evaluated a subset of the confirmed genes, as well as additional genes with related biological activities, by immunohistochemistry and laser capture microdissection of tumor sections, and their regulation in better-defined in vitro systems is now under investigation.

Herrmann, John

[10]

\section{A genomics approach to therapeutic antibody target discovery}

John Herrmann, Luca Rastelli, Elma Fernandes, Catherine Burgess, William LaRochelle, Henri Lichenstein \& Rick Shimkets

CuraGen Corporation, 555 Long Wharf Drive, New Haven, Connecticut 06511, USA

Functional genomics is expected to bring about significant advances in the discovery and development of new anticancer agents. Genomics-based drug target discovery will revolutionize how cancer is detected and classified, resulting in more finely tailored therapies. The explosion of information generated by large-scale functional genomics technologies has led to an exponential increase in the number of potential genes and proteins available for pharmaceutical and diagnostic research development. In tapping this potential, the primary challenge is to develop a strategy to integrate and extract meaning from the human genomic sequence information generated since the start of the Human Genome Project, relying on pragmatic strategies to sort and triage this information. Using a suite of integrated, high-throughput genomics technologies developed at CuraGen, we have devised streamlined approaches to identify new gene sequences rapidly, link them to specific diseases using reverse biology and finally validate them as effectors of disease progression. Application of these technologies has enabled us to identify 24 antibody drug targets for further evaluation and possible development as anticancer agents. We will discuss the role of some of the targets in cancer progression.

Hester, Susan

[11]

\section{Studies of normal gene expression in the rat nasal epithelium using cDNA array technology}

Susan Hester ${ }^{1,2}$, Gina Benavides ${ }^{3}$, Maureen Sartor ${ }^{3}$, Lawrence Yoon $^{3}$, Kevin Morgan ${ }^{3} \&$ Douglas Wolf ${ }^{1}$

${ }^{1}$ United States Environmental Protection Agency, Durham, North Carolina, USA ${ }^{2}$ Department of Pathology, University of North Carolina at Chapel Hill, Chapel Hill, North Carolina, USA

${ }^{3}$ Glaxo Wellcome Pharmaceutical Company, Research Triangle Park, North Carolina, USA

The nasal epithelium is an important target site for chemically induced toxicity and carcinogenicity. Gene expression data are being used increasingly in studies of such conditions. In order to provide normal baseline data for this target organ, we investigated gene expression profiles in nasal transitional and respiratory epitheli- 


\section{Abstracts: Session II}

um of healthy rats. We collected cells lining the anterior nasal passages, as confirmed by light microscopy, into $200 \mu \mathrm{l}$ of Trizol reagent by controlled intranasal instillation. We performed gene expression analysis using Clontech complementary DNA Rat Atlas 1.2 arrays (approximately 1,200 genes) and verified selected transcripts using the polymerase chain reaction with reverse transcription (Taqman). The percentages of genes within specific average expression ranges, on the basis of phosphorimager signal intensities, were $1.4 \%$ at $3,000-45,000,3.3 \%$ at $1,000-3,000,30.1 \%$ at $100-1,000$, and $65.2 \%$ below 100 . The most highly expressed genes included those involved in phase I (for example, cytochrome Ps) and phase II (for example, glutathione $S$-transferases) xenobiotic metabolism, bioenergetics (for example, cytochrome oxidase), osmotic balance (for example $\left(\mathrm{Na}^{+}+\mathrm{K}^{+}\right)$ATPase) and epithelial ionic homeostasis (for example, ion channels). These findings are consistent with normal nasal epithelial functions. Such baseline data will contribute to a further understanding of the functions of this region of the respiratory tract and facilitate the interpretation of treatment- or disease-related responses by the nasal epithelial transcriptome.

\section{Holzmann, Klaus}

\section{Kinetics of expression analysis during myc- mediated apoptosis}

Klaus Holzmann, Chantal Schamberger, Gerlinde Schmidt, Barbara Skrzypek, Soleman Sasgary \& Christa Cerni

Institute of Cancer Research, University of Vienna, Borschkegasse 8a, A-1090 Vienna, Austria

Malignant transformation in vivo as well as in vitro requires the activation of oncogenes concomitant with the inactivation of tumor suppressor genes. Although data on the activities and functions of single oncogenes such as c-myc and c-Haras are abundant, less information is available on their synergistic action. We found that primary rat embryo cell lines transformed by c-myc and c-Ha-ras were prone to apoptosis after inhibition of MEK, a downstream kinase of the main Ras signaling pathway. Addition of the MEK1 inhibitor U0126 to a series of myc/rasexpressing cell lines uniformly resulted in unphosphorylated ERK1 and ERK2, morphological alterations and cell cycle arrest, and apoptosis occurred to various extents. The MR- 6 cell line apoptosed most promptly after inhibition of MEK1. We analyzed in detail the kinetics of gene regulation of MR-6 cells during apoptosis by means of complementary DNA arrays for the expression of 1,200 characterized rat genes. A twofold increase in expression or reduction of expression below $50 \%$ was chosen as the threshold. More than 100 genes were found to be regulated during the early stage of apoptosis induction. One hour after addition of U0126, numerous growth factor receptor genes were induced. By $3 \mathrm{~h}, \mathrm{G1}$ - or Sphase-specific genes, genes of the AP-1 transcription factor complex and genes coding for cell-surface antigens were upregulated. By $6 \mathrm{~h}$, when MR- 6 cells started to apoptose, the expression of most of them returned to baseline values. Data obtained with cDNA arrays were confirmed by northern and western blot analysis. The expression pattern and kinetics of gene regulation in U0126-treated MR6 cells were compared with those of the MR-2 cell line, in which inhibition of MEK1 resulted in G1 arrest but not cell death. We conclude that myc-mediated apoptosis is accompanied by a transient wave of upregulation of a complex array of genes. At least some of them might represent an abortive attempt by cells to avert cell death.
Huang, Kuan-Chun

[13]

\section{Global RNA expression analysis of primary and recurrent ovarian tumors}

\author{
Kuan-Chun Huang, Samuel Mok, Ross Berkowitz \& Ng Shu- \\ Wing
}

Brigham and Women's Hospital, Harvard Medical School, Boston, Massachusetts, USA

Many antineoplastic agents fail because of intrinsic or acquired resistance developed by the cancer cells; drug resistance is therefore a major obstacle to successful chemotherapy of human cancers, including ovarian cancer. Understanding the mechanisms by which drug resistance arises and identification of the molecular factors affecting new drug target development is of paramount importance. In a study to compare the global RNA expression profiles of primary and recurrent ovarian tumors from the same patient by means of oligonucleotide microarray analysis, we have identified XIST (inactive X [Xi] chromosome-specific transcripts) as the most differentially expressed gene that was downregulated in the recurrent tumor. The XIST gene resides within the $\mathrm{X}$ inactivation center and is unique in being expressed exclusively from the inactive X chromosome. XIST is both necessary and sufficient for X inactivation. Preliminary studies of the cell line derived from the recurrent tumor showed that the line is resistant to the antineoplastic agent taxol, one of the anticancer drugs included in the treatment of the patients under study. The recurrent cell line has at least one copy of XIST in the genome, although the gene is not expressed. Further characterization of XIST expression in a panel of ten ovarian cell lines and six breast cancer cell lines showed that the expression levels of XIST correlate significantly with taxol sensitivity. These data indicate a possible correlation between $\mathrm{X}$ inactivation and taxol resistance in ovarian cancer. We have launched a detailed mechanistic study to characterize the underlying mechanism.

Huang, Tim Hui-Ming

[14]

\section{Dissecting complex genetic and epigenetic alterations in cancer genomes using CpG island microarrays}

\author{
Tim Hui-Ming Huang, Huidong Shi, Farah Rahmatpanah \\ $\&$ Pearlly Yan
}

Department of Pathology and Anatomical Sciences, Ellis Fischel Cancer Center, University of Missouri-Columbia, Columbia, Missouri 65203, USA

We describe a high-throughput approach for investigating both copy number alterations and aberrant methylation of $\mathrm{CpG}$ islands in human cancer. The technique involves generation of a microarray panel of 5,150 short GC-rich tags $(0.2-2$ kilobases) derived from a CpG island genomic library. This DNA chip was used to study a patient with invasive breast cancer. We first restricted tumor and normal DNA from the patient with a four-base frequent cutter known to preserve larger GC-rich CpG island fragments. The digests were ligated to linkers for the polymerase chain reaction in low-amplification cycles, allowing for semiquantitative analysis in subsequent microarray hybridization. We co-hybridized amplicons from test and reference samples in a two-color fluorescence system, similar to that of the comparative genomic hybridization. The differential signal intensities observed in the microarray panel reflect copy number alterations of CpG islands in the tumor DNA. For methylation analysis, we further digested the ligated DNA with methylation-sensitive restriction enzymes and amplified it using the linker polymerase chain reaction. The amplified products may therefore contain different pools of DNA fragments owing to the differential methylation status of tumor relative to the normal control. We detected these methylation differences in subse- 